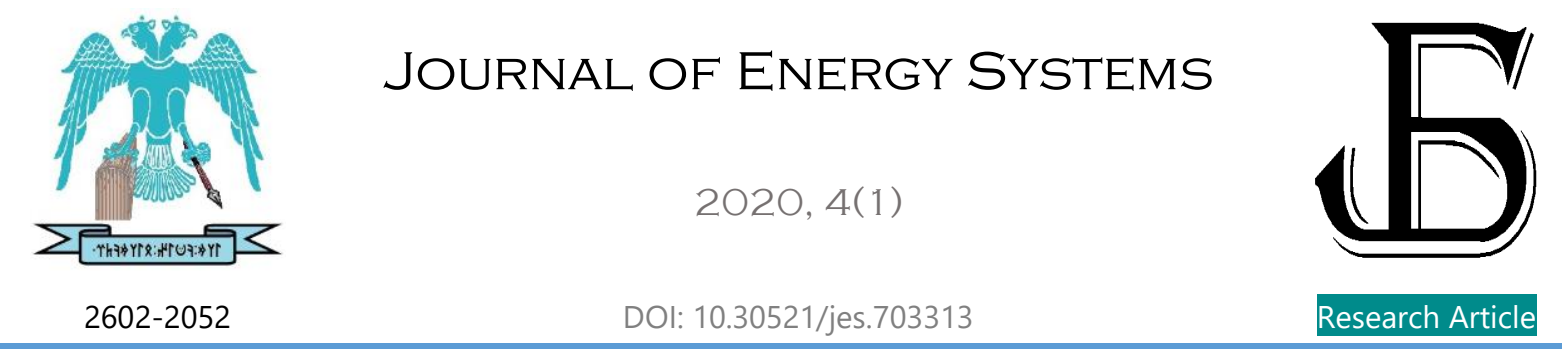

\title{
Neutron yield and ion production with respect to cathode radius in spherical plasma focus
}

\author{
Yaşar Ay \\ Dicle University, Department of Electrical and Electronics Engineering, 21280, Diyarbakır, Turkey, \\ yasar.ay@dicle.edu.tr
}

\begin{abstract}
Submitted: $\quad$ 13.03.2020
Accepted: 28.03 .2020

Published: $\quad 31.03 .2020$

Abstract: The effect of the cathode radius variation (from $11.5 \mathrm{~cm}$ to $17 \mathrm{~cm}$ ) on neutron yield, discharge current, plasma temperature, and ion properties (velocity, energy, and density) are investigated in this study using the developed spherical plasma focus model and the results are reported in this paper. Peak discharge current and peak beam-ion properties decrease with increasing the cathode radius. Maximum plasma temperature $(22.34 \mathrm{eV})$ and maximum beam-target neutron yield $\left(1.18 \times 10^{13}\right)$ are achieved using the cathode with $15 \mathrm{~cm}$ radius. The longest pinch duration for all calculations is also achieved using $15 \mathrm{~cm}$ cathode radius. It is found that the optimum cathode radius is $15 \mathrm{~cm}$ in terms of the neutron yield, plasma temperature and beam-ion properties in spherical plasma focus device.
\end{abstract}

Keywords: Beam-ion, Cathode, Neutron yield, Plasma focus

Cite this paper as: Ay, Y., Neutron yield and ion production with respect to cathode radius in spherical plasma focus. Journal of Energy Systems 2020; 4(1): 22-31, DOI: 10.30521/jes.703313

(c) 2020 Published by peer-reviewed open access scientific journal, JES at DergiPark (https://dergipark.org.tr/en/pub/jes) 


\section{INTRODUCTION}

Plasma focus (PF) as pulsed devices are used to produce the pinch, which is hot and dense plasma. Pinch is produced between electrodes by gas discharge. Electron and ion beams can also be accelerated to high energies by plasma focus devices to produce neutrons, ions, and x-rays using deuterium (D), tritium (T), or a mixture of D-T gases.

The optimum length and shape of the plasma focus electrodes are investigated to see their effects on the plasma dynamics so that the optimized plasma focus devices can be built in terms of the electrode length and radius in addition to the optimum electrode shapes to produce higher neutrons, $\mathrm{x}$-rays, or ions efficiently [1-6]. While Hill and Hubs [1] decreased waist diameter of the cathode to improve neutron yield, Wong et al. [2] used tapered anode for x-ray investigation. Borthakur et al. [3] studied the effect of the electrode's diameters and separation on detection efficiency of vacuum photodiodes in plasma focus device. Two different cathodes were designed and tested [4] for x-ray yield and it is found that cathode design and electrode separation have considerable effect on the x-ray yield. Hayati et al. [5] used a different approach in that they used artificial neural network to optimize anode shape for higher $\mathrm{x}$-ray yield.

Thermonuclear and beam-target neutron production mechanisms are the two widely accepted ways to produce neutrons in plasma focus devices. While interaction of the thermal deuterons under maximum compression produces thermal neutrons (thermonuclear neutron production), beam-target neutrons are produced through fusion reactions with the accelerated ion beams that interact with thermal deuterons under diode voltage. Neutron yield and energy of neutrons are anisotropic which leads to the dominant beam-target neutron production compared to thermonuclear neutron production in plasma focus devices, which is also the case in this study with spherical plasma focus device [6-8].

Tubular and squirrel cage cathodes are mostly used cathode designs in plasma focus devices. Both of these designs have their advantages and disadvantages. Smaller plasma focus devices can be build using tubular cathode but plasma focus with tubular cathode produces less pinch current compared to squirrel cage design. Impurities is also increased if tubular cathode is used in PF devices. Increased impurities cause radiation loss which is also the reason of less neutron production in tubular cathodes compared to squirrel cage cathodes. Changing cathode design from tubular to squirrel cage results in better neutron production. Squirrel cage cathode compose of a number of rods which is one of important design parameters to optimize plasma focus such that when the number of rods in squirrel cage cathode are decreased, the impurities and instabilities are increased because of the runaway electrons in addition to slower movement of Current Sheath (CS). In addition to type of cathode designs, the radius of the cathode also influences the CS dynamics and instabilities such that large energy densities and intense radiations result from a cathode with a small radius, and slower plasma movement is due to a cathode with a large radius $[9,10]$.

A miniature plasma focus device is used with two different cathode designs, which are tubular and squirrel cage cathodes, to investigate the neutron yield. While tubular cathode produced $1.82 \times 10^{5}$ neutrons per shot at 4 mbar, squirrel cage cathode produced $1.15 \times 10^{6}$ neutrons per shot at 6 mbar. It can be seen that it is possible to increase neutron yield by using squirrel cage cathode in plasma focus devices [11].

In addition to the cathode design, anode length is also an important parameter for neutron yield. Increasing anode length increases neutron yield until some optimum anode length. Beyond this optimum point, increasing anode length results in decreasing neutron yield. When anode length is too short, the current sheath will not reach sufficient velocity for good focus action. Similarly, when anode length is too large, the current sheath become unstable for focusing. Therefore, departure from optimum anode length leads to a decrease in neutron yield due to poor focusing [12]. 
In the previous works, a model for spherical plasma focus (SPF) is developed and validated [13,14], spherical plasma focus device is optimized for neutron yield [15] and the effect of spherical cathode radius on plasma dynamics is studied [16]. Now this cathode variation effect is investigated for neutron yield and ion properties in this study.

The main objective here is to investigate the cathode radius effect (ranging from $11.5 \mathrm{~cm}$ to $17 \mathrm{~cm}$ with $0.5 \mathrm{~cm}$ increment) on neutron yield, plasma temperature, discharge current, and beam-ion properties (velocity, energy and density) in SPF device using developed SPF model [13,14].

\section{SPHERICAL PLASMA FOCUS MODEL}

Since the developed model and model validation are given in detail in previous works $[13,14]$, a brief description of model is mentioned in this paper.

Modeled spherical plasma focus device has two concentric electrodes. The developed model uses shock wave equations and snow plow model and couple them with equivalent circuit model to describe SPF device $[13,14]$. The model has 4 phases which are rundown phase I, rundown phase II, reflected shock phase, and radiative phase.

Gas discharge between electrodes creates the current sheath between electrodes which is the beginning of rundown phase I. This phase ends and the second phase (rundown phase II) starts when CS reaches equator point of device. Then shock front hits axis of device which starts reflected shock phase. Then shock front reflects towards CS. This phase then ends and the last phase (radiative phase) begins when reflected shock front reaches the coming CS. Compression of plasma column in this phase continues until the maximum compression. At this point in the SPF, plasma disruption occurs and ends radiative phase.

Figure 1 shows configuration and equivalent circuit model for the SPF. In the circuit model, $V_{0}$ is charging voltage, $C_{0}$ represents the capacitor bank. $L_{0}$ and $r_{0}$ represent circuit inductance and resistance. $L_{p}$ and $r_{p}$ represent the plasma inductance and resistance.

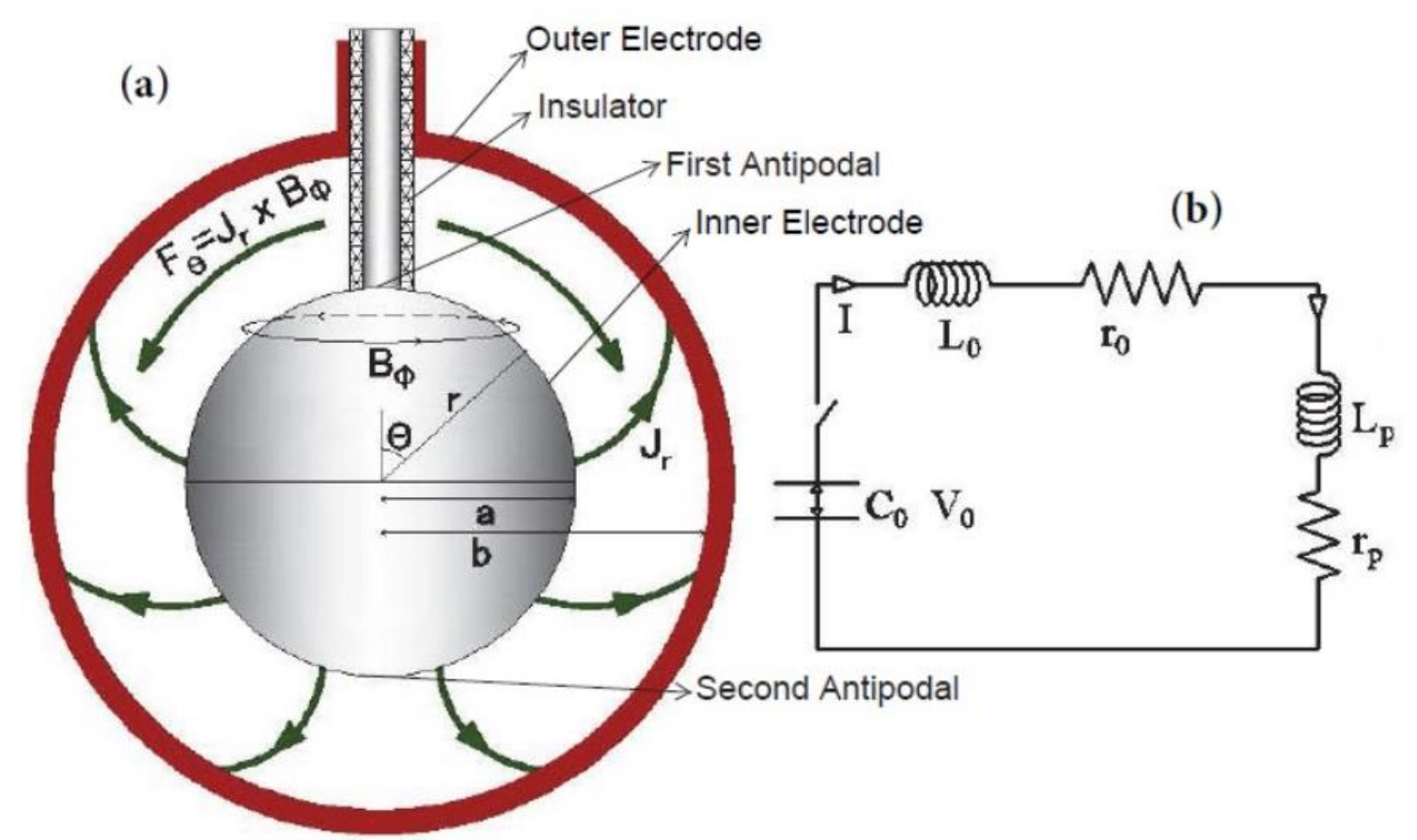

Figure 1. (a) Spherical plasma focus device configuration (b) Equivalent circuit model of SPF 


\subsection{Governing Equations}

Derivation of the model equations is explained and given in the previous works [13, 14]. Therefore, only the final equations with brief explanations are given here.

The magnetic pressure on the CS results in a change in the momentum of the CS. Therefore, this momentum change is set equal to the magnetic pressure to form a balance equation which is then solved for $\ddot{\theta}$ that is the equation of motion for the CS. This equation of motion is calculated for all phases as follows $[13,14]$ :

Equation of motion for rundown phase I:

$$
\ddot{\theta}=\frac{\alpha^{2} I^{2}}{r \sin \theta\left(\cos \theta_{0}-\cos \theta\right)}-\frac{\dot{\theta}^{2} \sin \theta}{\cos \theta_{0}-\cos \theta}
$$

Equation of motion for rundown phase II and reflected phase:

$$
\ddot{\theta}=\frac{\alpha^{2} I^{2}}{r \cos (\theta-\pi / 2)\left(\cos \theta_{0}-\cos \theta\right)}-\frac{\dot{\theta}^{2} \sin \theta}{\cos \theta_{0}-\cos \theta}
$$

where $\alpha=\frac{3 \mu_{0} f_{c}^{2} \ln \left(\frac{b}{a}\right)}{8 \pi^{2} \rho f_{m}\left(b^{3}-a^{3}\right)}$ is a scaling parameters that consist of constant values. $I$ is discharge current, $r$ is the distance for plasma parameter calculations, $\theta$ is angle of the motion, $\theta_{0}$ is an angle corresponding to the insulator volume, $\mu_{0}$ is permeability of free space, $f_{c}$ and $f_{m}$ are current and mass fraction, a is inner and $b$ is outer electrode radius, $\rho$ represents initial gas density.

For the last phase (radiative phase), radiation terms are taken into account and there is a third force on the CS due to radiation emissions. In this phase, the momentum change of the current sheath is because of the magnetic pressure on the current sheath and radiation emission, which leads to the following equation as the equation of the motion for radiative phase:

$$
\ddot{\theta}=\frac{\alpha^{2} I^{2}}{r \cos (\theta-\pi / 2)}-\frac{\sin \theta \dot{\theta}^{2}}{C}-\frac{3 Q A}{2 \pi \rho f_{m}\left(b^{3}-a^{3}\right) r C}
$$

where $C=\cos \theta_{0}-\cos \theta$ and $\mathrm{A}$ is plasma column surface area.

Equation for neutron yield:

$$
Y=N_{b} N_{i} V_{c o l} \sigma v_{b}
$$

where $N_{b}$ and $N_{i}$ are beam-ion number density and plasma-ion number density, $V_{c o l}$ is the pinch volume, $\sigma$ is cross section for DT reaction, $v_{b}$ is beam-ion velocity.

Plasma-ion number density:

$$
N_{i}=N_{0} f_{m} \frac{2}{1+\cos \theta}
$$

Beam-ion number density:

$$
N_{b}=\frac{f_{i} L I^{2} f_{c}^{2}}{2 e U V_{c o l}}
$$


Where $f_{i}$ represents how much pinch inductive energy is converted into beam kinetic energy, $N_{o}$ is ambient gas number density, $e$ is the elementary charge, and $U$ is diode voltage, $L$ is plasma inductance.

\section{RESULTS AND DISCUSSION}

In this work, a spherical plasma focus with $432 \mu \mathrm{F}$ capacitor bank energy is simulated using $25 \mathrm{kV}$ charging voltage, 14.3 Torr D-T gas pressure (equal amount of D-T mixture) and $36 \mathrm{nH}$ external inductance. The inner electrode (anode) radius of the SPF device is $8 \mathrm{~cm}$. The outer electrode (cathode) radius is changed from 11.5 to $17 \mathrm{~cm}$ using $0.5 \mathrm{~cm}$ increment so that its effect on neutron yield and ions can be investigated. The lines in the figures below are also plotted with the same order.

While figure 2 [16] shows the discharge currents for the selected cathode radii, figure 3 shows the maximum (peak) currents and dip discharge currents with respect to cathode radius variation. In figure 2 , only 4 cathode radii are plotted so that the lines can be seen clearly but the change of the discharge currents with respect to cathode radius can be seen clearly both in figure 2 and figure 3 . Discharge current for $11.5 \mathrm{~cm}$ cathode radius reaches the maximum value of $1508 \mathrm{kA}$. Increasing cathode radius results in decreasing both peak currents and dip currents in spherical plasma focus device.

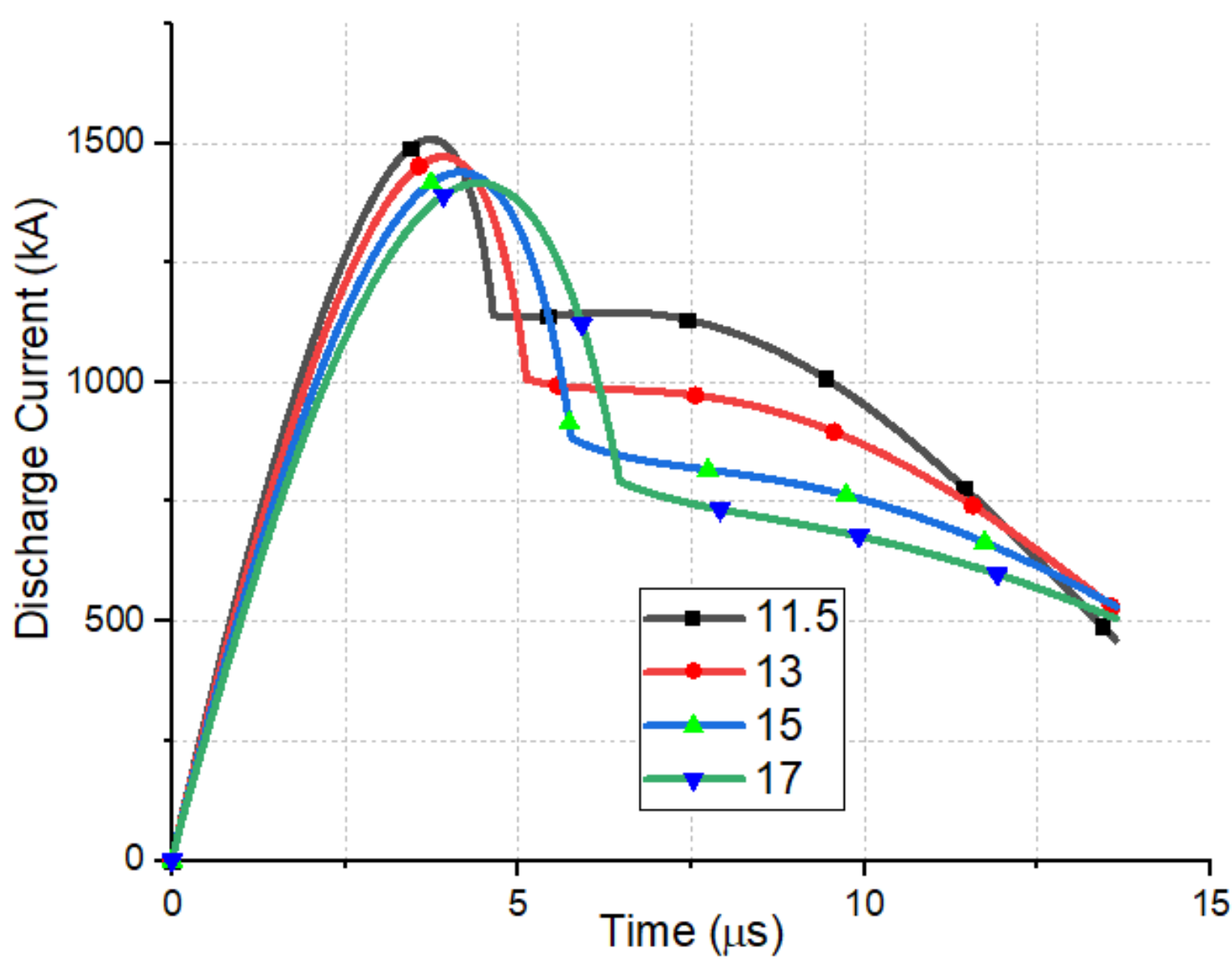

Figure 2. Discharge currents for different cathode radii [16] 


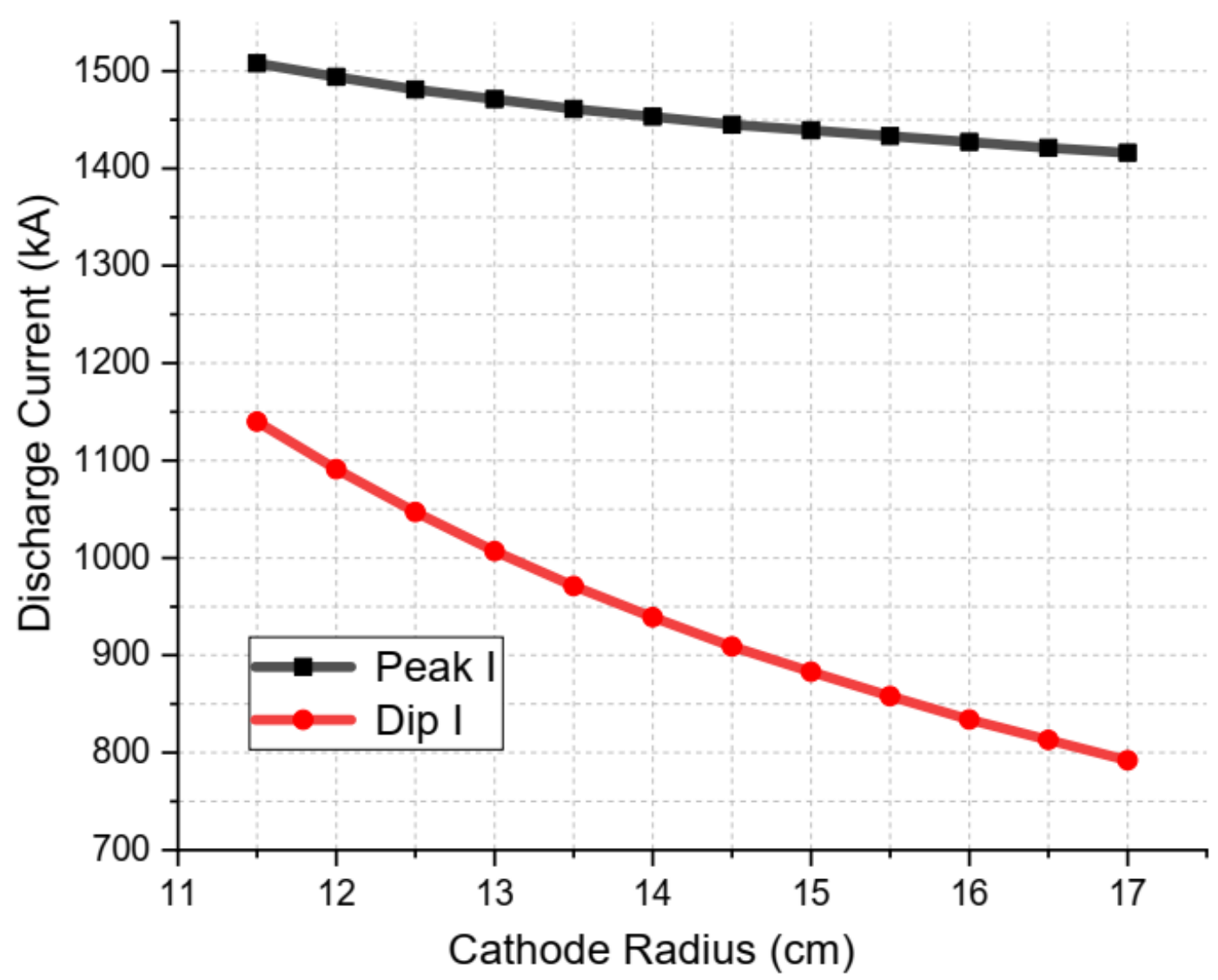

Figure 3. Peak and dip discharge currents with respect to cathode radius in the spherical plasma focus device

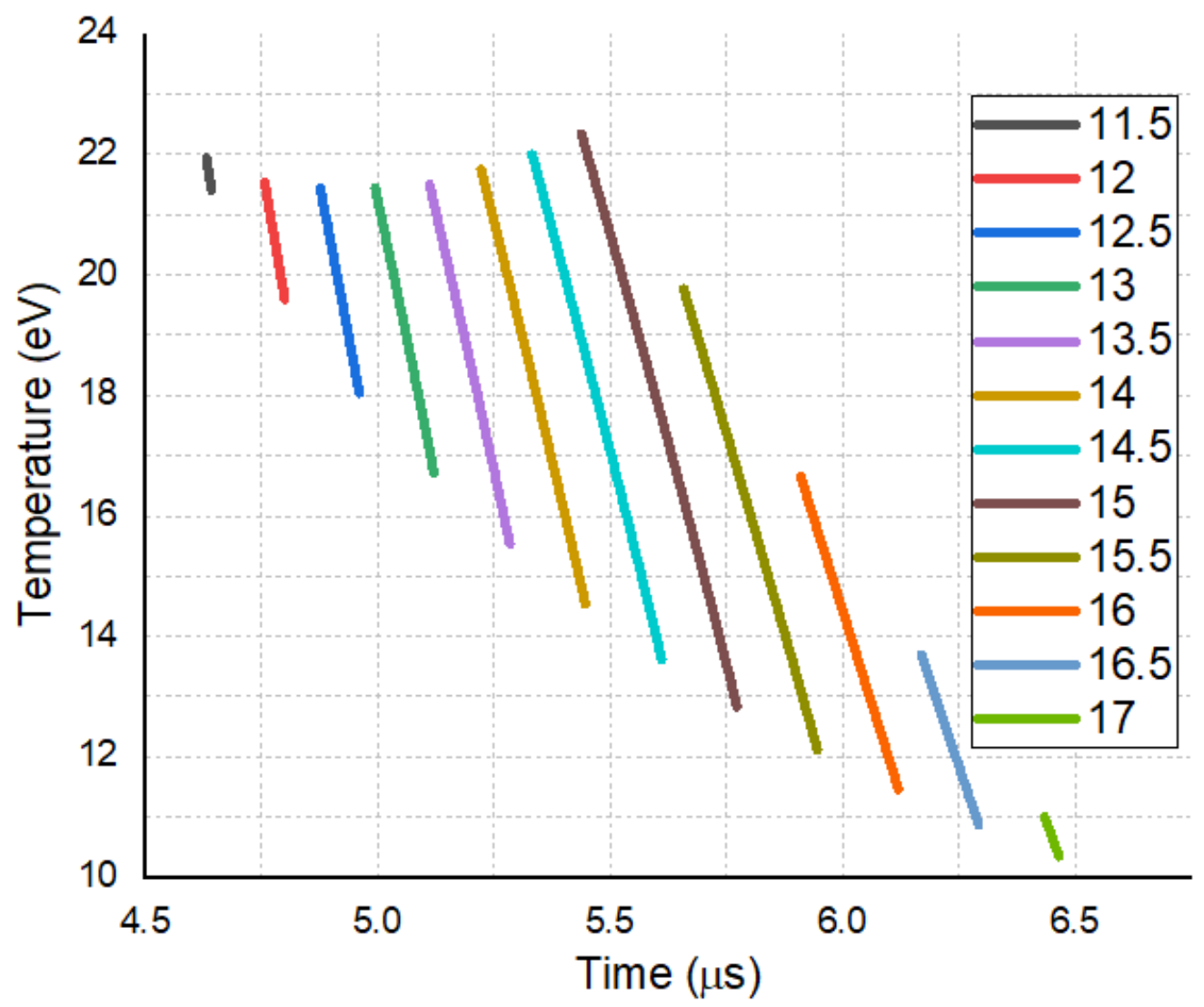

Figure 4. Plasma temperature for various cathode radii 
The cathode variation has different effect on plasma temperature which can be seen in figure 4 . Increasing cathode radius from $11.5 \mathrm{~cm}$ to $13 \mathrm{~cm}$ decreases the peak plasma temperature but then peak plasma temperature starts to increase with increasing the cathode radius from $13 \mathrm{~cm}$ to $15 \mathrm{~cm}$. Plasma temperature reaches the peak value of $22.34 \mathrm{eV}$ using a cathode with $15 \mathrm{~cm}$ radius. Increasing the cathode radius beyond $15 \mathrm{~cm}$ causes plasma temperature to drop suddenly. Therefore, it can be deduced that $15 \mathrm{~cm}$ cathode radius is optimum cathode radius in terms of temperature.

Figure 5 and figure 6 show beam-ion velocity and corresponding beam-ion energy. Beam-ions are accelerated under diode voltage. Since increasing the cathode radius decreases the discharge current which results in a decrease in diode voltage, increasing the cathode radius decreases the beam-ion velocity and beam-ion energy.

Even though maximum beam-ion velocity $(226.95 \mathrm{~cm} / \mu \mathrm{s})$ and beam-ion energy $(53.79 \mathrm{keV})$ are obtained using cathode with $11.5 \mathrm{~cm}$ radius, a cathode with $15 \mathrm{~cm}$ radius has longer pinch duration which makes $15 \mathrm{~cm}$ cathode radius the optimum cathode in terms of the beam-ion velocity and beamion energy. Increasing cathode radius beyond $15 \mathrm{~cm}$ results in short pinch duration with lower beamion velocity and energy. Maximum beam-ion velocity and beam-ion energy for the cathode with $15 \mathrm{~cm}$ radius are $207.39 \mathrm{~cm} / \mu \mathrm{s}$ and $44.92 \mathrm{keV}$.

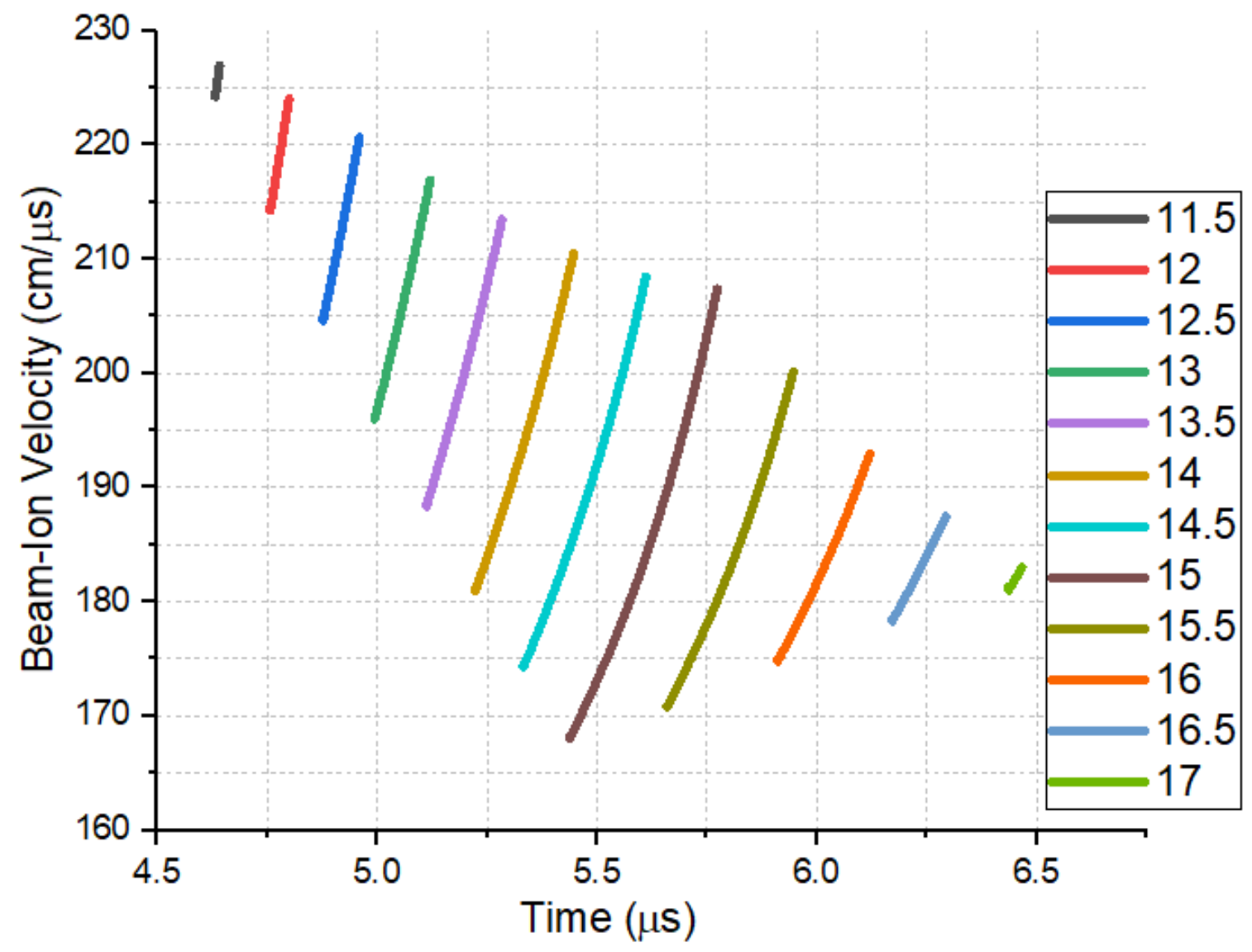

Figure 5. Beam-ion velocity for various cathode radii 


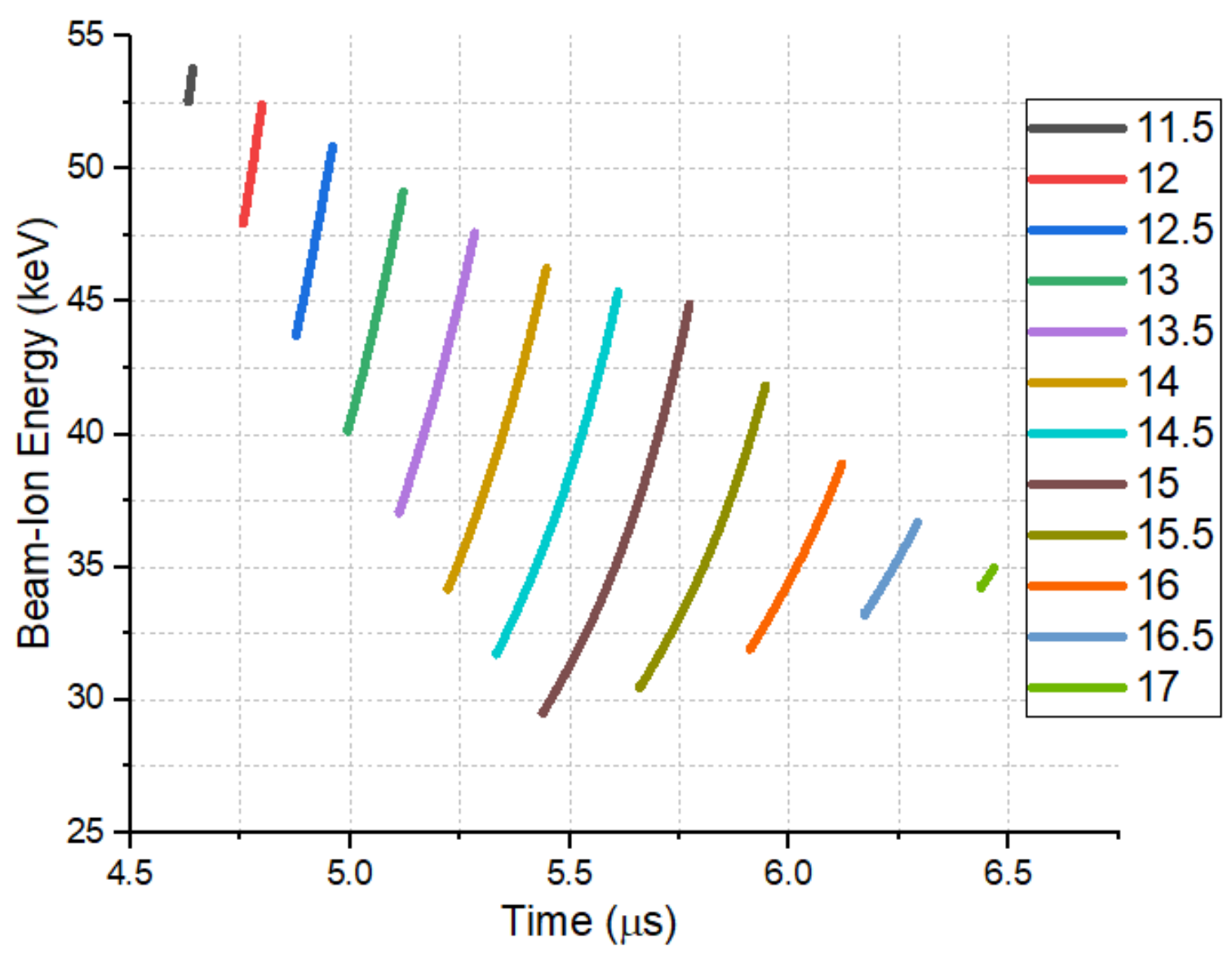

Figure 6. Beam-ion energy for various cathode radii

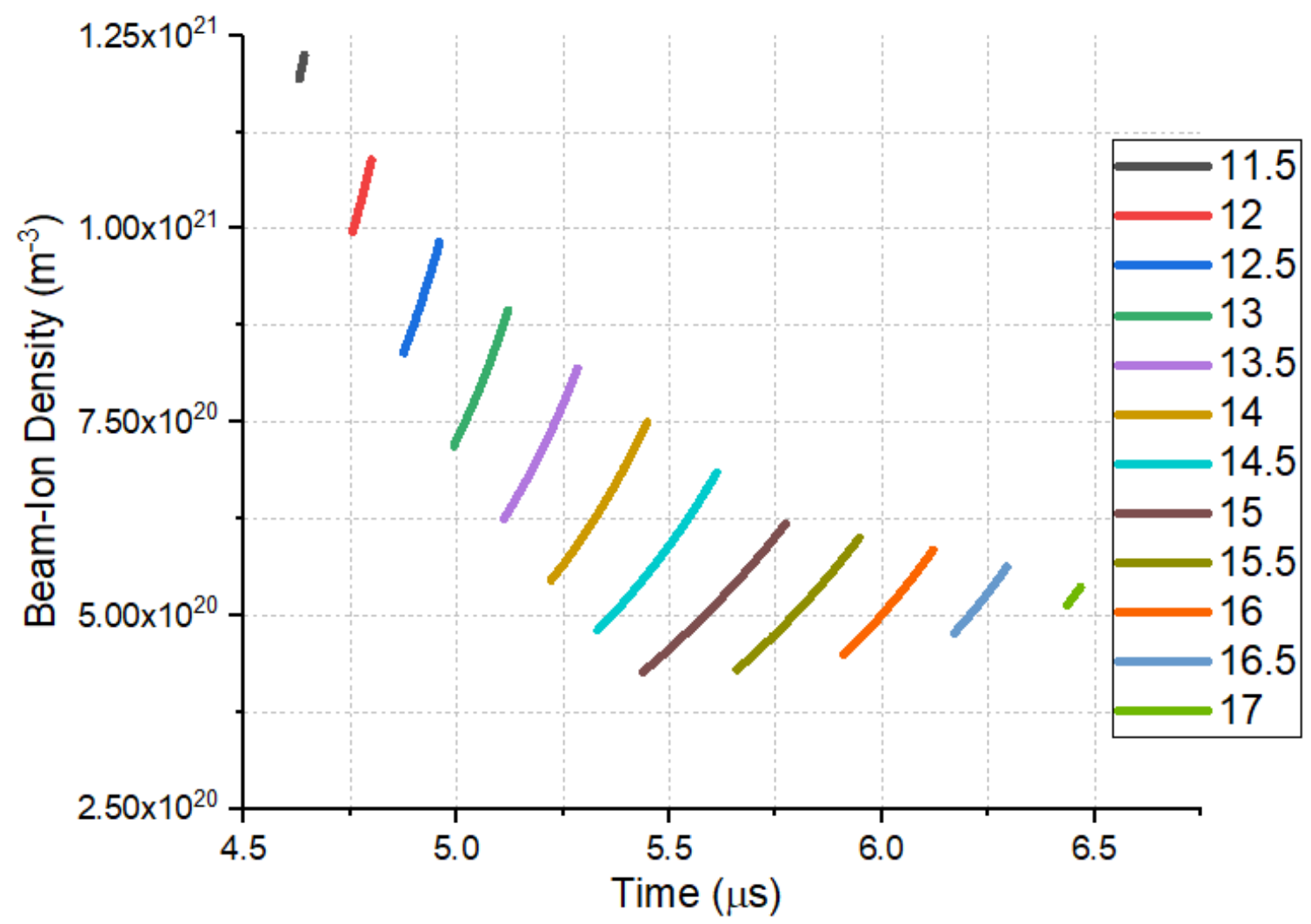

Figure 7. Beam-ion density for various cathode radii 
The effect of cathode radius variation on beam-ion density (figure 7) is similar to beam-ion velocity and beam-ion energy. Beam-ion density reaches the maximum of $1.23 \times 10^{21} \mathrm{~m}^{-3}$ using $11.5 \mathrm{~cm}$ cathode radius. Beam-ion density decreases with increasing cathode radius. Rate of beam-ion density decrease starts to slow down after $15 \mathrm{~cm}$ cathode radius and pinch duration is the longest using the cathode with $15 \mathrm{~cm}$ radius. The maximum beam-ion density for $15 \mathrm{~cm}$ cathode radius is $6.19 \times 10^{20} \mathrm{~m}^{-3}$.

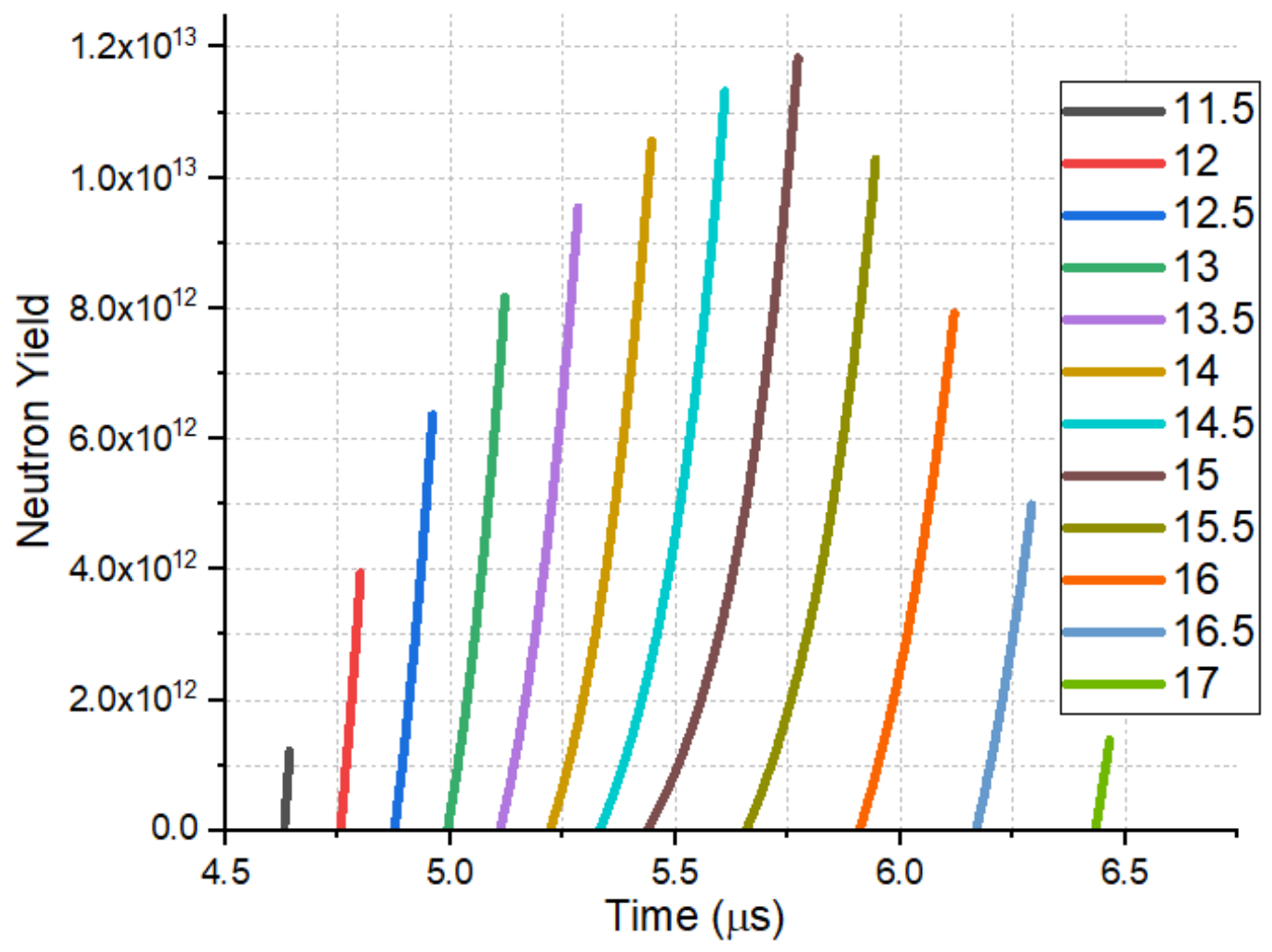

Figure 8. Beam-target neutron yield for various cathode radii

Figure 8 shows the beam-target neutron yield in SPF device. Increasing cathode radius starts to increase neutron yield until the cathode with $15 \mathrm{~cm}$ radius. Increasing the cathode radius beyond $15 \mathrm{~cm}$ results in decreasing the neutron yield. It can be concluded that $15 \mathrm{~cm}$ cathode radius is the optimum cathode radius in terms of beam-target neutron production. Beam-target neutron yield reaches maximum value of $1.18 \times 10^{13}$ neutrons for $15 \mathrm{~cm}$ cathode radius. It also has longest pinch duration.

\section{CONCLUSION}

A spherical plasma focus device is simulated in this work with different cathode radii ranging from 11.5 $\mathrm{cm}$ to $17 \mathrm{~cm}$. It is found that increasing the cathode radius decreases the discharge current, beam-ion velocity, beam-ion energy and beam-ion density but maximum plasma temperature and beam-target neutron yield is obtained using the cathode with $15 \mathrm{~cm}$ radius. The longest pinch duration is also obtained with $15 \mathrm{~cm}$ cathode radius for all calculations. In this study, neutron yield of $1.18 \times 10^{13}$ and plasma temperature of $22.34 \mathrm{eV}$ are calculated with $15 \mathrm{~cm}$ cathode radius. Considering all these calculations, it can be concluded that the cathode with $15 \mathrm{~cm}$ radius is the optimum value for SPF under given conditions.

\section{Acknowledgment}

This work was supported by The Scientific and Technological Research Council of Turkey, Project No: 119F039 


\section{REFERENCES}

[1] Hill, R., Hubbs, J., A multi-shot plasma focus with improved cathode design, Physics Letters A, 1983, 98(8), 417-420.

[2] Wong, D., Lee, P., Zhang, T., Patran, A., Tan, T., Rawat, R., Lee, S., An improved radiative plasma focus model calibrated for neon-filled nx2 using a tapered anode, Plasma Sources Science and Technology, 2006, 16(1), 116.

[3] Borthakur, T., Talukdar, N., Neog, N., Rao, C., Shyam, A., Detection efficiency vs. cathode and anode separation in cylindrical vacuum photodiodes used for measuring x-rays from plasma focus device, Review of Scientific Instruments, 2011, 82(10), 103507.

[4] Khan, M.Z., Yap, S.L., Khan, M.A., Rehman, A., Zakaullah M., Effect of cathode designs on radiation emission of compact diode (cd) device, Journal of Fusion Energy, 2013, 32(1), 34-41.

[5] Hayati, M., Roshani, G., Abdi, H., Rezaei, A., Mahtab, M., An optimized design of anode shape based on artificial neural network for achieving highest x-ray yield in plasma focus device, Journal of Fusion Energy, 2013, 32(6), 615-621.

[6] Kubes, P., Paduch, M., Sadowski, M., Cikhardt, J., Cikhardtova, B., Features of fast deuterons emitted from plasma focus discharges, Physics of Plasmas, 2019, 26(3), 032702.

[7] Talebitaher, A., Springham, S.V., Rawat, R.S., Lee, P., Plasma focus neutron anisotropy measurements and influence of a deuteron beam obstacle, Nuclear Instruments and Methods in Physics Research Section A: Accelerators, Spectrometers, Detectors and Associated Equipment, 2017, 848, 60-65.

[8] Lim, L.K., Yap, S.L., Bradley, D., Time-resolved characteristics of deuteron beam generated by plasma focus discharge, Plos One, 2018, 13(1), e0188009.

[9] Gourdain, P.A., Greenly, J.B., Hammer, D.A., Knapp, P.F., Kusse, B.R., The impact of cathode diameter on radial foil explosions, IEEE Transactions on Plasma Science, 2010, 38(12), 3363-3369.

[10] Piriaei, D., Javadi, S., Mahabadi, T.D., Yousefi, H.R., Elahi, A.S., The influence of the cathode array and the pressure variations on the current sheath dynamics of a small plasma focus device in the presence of an axial magnetic probe, Physics of Plasmas, 2017, 24(4), 043504.

[11] Verma, R., Rawat, R.S., Lee, P., Lee, S., Springham, S.V., Tan, T.L., Krishnan, M., Effect of cathode structure on neutron yield performance of a miniature plasma focus device, Physics Letters A, 2009, 373, 2568-2571.

[12] Beg, F.N., Zakaullah, M., Nisar, M., Murtaza, G., Role of anode length in a mather-type plasma focus, Modern Physics Letters B, 1992, 6(10), 593-597.

[13] Ay, Y., Al-Halim, M.A., Bourham, M.A., Simulation of the plasma sheath dynamics in a spherical plasma focus, The European Physical Journal D, 2015, 69(9).

[14] Ay, Y., Al-Halim, M.A., Bourham, M.A., MHD simulation for neutron yield, radiations and beam-ion properties in the spherical plasma focus, Journal of Fusion Energy, 2015, 35(2), 407-414.

[15] Ay, Y., A neutron source with 1014 DT neutron yield, International Journal of Modern Physics E, 2020, 28(11), 1950097.

[16] Ay, Y., Effect of the cathode radius on plasma dynamics and radiation emissions in a spherical plasma focus device, Physics of Plasmas, 2019, 26(10), 102506. 